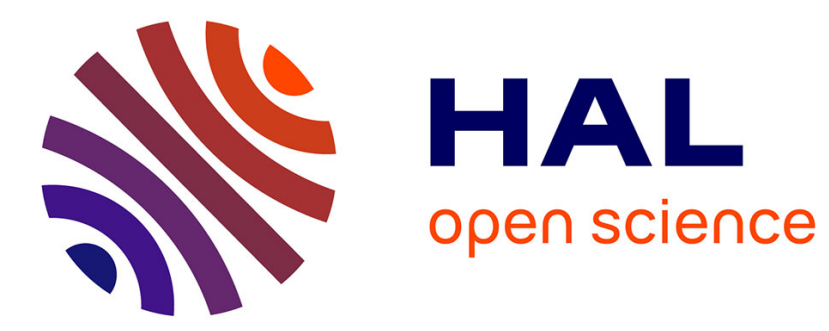

\title{
The Maximum of a Random Walk and Its Application to Rectangle Packing
}

\author{
E.G. Coffman, Philippe Flajolet, Leopold Flatto, Micha Hofri
}

\section{To cite this version:}

E.G. Coffman, Philippe Flajolet, Leopold Flatto, Micha Hofri. The Maximum of a Random Walk and Its Application to Rectangle Packing. [Research Report] RR-3223, INRIA. 1997. inria-00073466

\section{HAL Id: inria-00073466 \\ https://hal.inria.fr/inria-00073466}

Submitted on 24 May 2006

HAL is a multi-disciplinary open access archive for the deposit and dissemination of scientific research documents, whether they are published or not. The documents may come from teaching and research institutions in France or abroad, or from public or private research centers.
L'archive ouverte pluridisciplinaire HAL, est destinée au dépôt et à la diffusion de documents scientifiques de niveau recherche, publiés ou non, émanant des établissements d'enseignement et de recherche français ou étrangers, des laboratoires publics ou privés. 


\section{The Maximum of a Random Walk and Its Application to Rectangle Packing}

E. G. Coffman, Philippe Flajolet, Leopold Flatto, Micha Hofri

N 3223

Juillet 1997

THÈME 2

\section{apport}

derecherche 



\title{
The Maximum of a Random Walk and Its Application to Rectangle Packing
}

E. G. Coffman, Philippe Flajolet, Leopold Flatto, Micha Hofri

Thème 2 - Génie logiciel

et calcul symbolique

Projet Algo

Rapport de recherche-N 3223- Juillet 1997 - 14 pages

\begin{abstract}
We consider a symmetric random walk of length $n$ that starts at the origin and takes steps uniformly distributed on the real interval $[-1,+1]$. We study the large- $n$ behavior of the expected maximum excursion and prove a very precise estimate. This estimate applies to the problem of packing $n$ rectangles into a unit-width strip; in particular, it makes much more precise the known upper bound on the expected minimum height when the rectangle sides are $2 n$ independent uniform random draws from $[0,1]$.
\end{abstract}




\section{Le maximum d'une marche aléatoire et son application au placement de rectangles}

Résumé : On considère une marche aléatoire partant de l'origine, de longueur $n$, et suivant des étapes uniformément distribuées dans l'intervalle $[-1,+1]$. Cet article étudie, pour $n$ grand, le comportement du maximum attendu de la marche aléatoire et fournit une estimation asymptotique précise de l'espérance. L'estimation s'applique alors au placement de $n$ rectangles dans une bande de largeur unité; en particulier elle permet de rendre beaucoup plus précise la borne supérieure sur la hauteur d'un tel placement lorsque les cotés des rectangles sont des variables aléatoires uniformément distribuées sur l'intervalle $[0,1]$. 


\title{
The Maximum of a Random Walk and Its Application to Rectangle Packing
}

\author{
E. G. Coffman ${ }^{1}$, Jr., Philippe Flajolet ${ }^{2,4}$, Leopold Flatto ${ }^{1}$, and Micha Hofri ${ }^{3}$
}

1. Bell Labs, Lucent Technologies, 700 Mountain Ave., Murray Hill, NJ 07974.

2. INRIA-Rocquencourt, F-78153 Le Chesnay, France.

3. Dept. of Computer Science, Rice University, Houston, TX 77005.

May 19, 1997

\begin{abstract}
Let $S_{0}, \ldots, S_{n}$ be a symmetric random walk that starts at the origin $\left(S_{0}=0\right)$, and takes steps uniformly distributed on $[-1,+1]$. We study the large- $n$ behavior of the expected maximum excursion and prove the estimate

$$
\mathrm{E} \max _{0 \leq k \leq n} S_{k}=\sqrt{\frac{2 n}{3 \pi}}-c+\frac{1}{5} \sqrt{\frac{2}{3 \pi}} n^{-1 / 2}+O\left(n^{-3 / 2}\right),
$$

where $c=0.297952 \ldots$. This estimate applies to the problem of packing $n$ rectangles into a unit-width strip; in particular, it makes much more precise the known upper bound on the expected minimum height, $\frac{n}{4}+\frac{1}{2} \mathrm{E} \max _{0 \leq j \leq n} S_{j}+\frac{1}{2}=\frac{n}{4}+O\left(n^{1 / 2}\right)$, when the rectangle sides are $2 n$ independent uniform random draws from $[0,1]$.
\end{abstract}

\section{Introduction}

We compute the large- $n$ behavior of the expected maximum of a symmetric random walk $S_{n}=\sum_{1 \leq k \leq n} Z_{k}, n \geq 0$, with the initial position $S_{0}:=0$, and with steps $Z_{k}$ drawn independently and uniformly at random from the interval $[-1,+1]$. Our result is applied to strip packings of rectangles and sharpens an expected-height estimate of Coffman and Shor [3] for rectangles with dimensions drawn independently and uniformly at random from $[0,1]$.

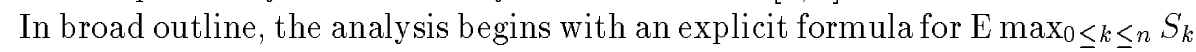
which involves an awkward combinatorial sum. The asymptotic analysis of this sum is approached via Rice's method, which in turn entails the asymptotics of the integral

$$
I_{n}:=\int_{0}^{\infty}\left[1-\prod_{j=1}^{n} \frac{x^{2}}{x^{2}+j^{2}}\right] d x .
$$

\footnotetext{
${ }^{4}$ The work of Philippe Flajolet was supported by the Long Term Research Project ALCOMIT (\# 20244) of the European Union.
} 
Interesting in its own right, the analysis of $I_{n}$ is given in Section 2, where we prove the following large- $n$ asymptotic behavior.

Theorem 1 For the integral in (1),

$$
I_{n}=\sqrt{\frac{\pi}{3}} n^{3 / 2}+\frac{7 \sqrt{3 \pi}}{40} n^{1 / 2}+O\left(n^{-1 / 2}\right) .
$$

Then Section 3 proves our main result.

Theorem 2 The expected maximum in $n$ steps of the random walk $S_{k}$ is

$$
\mathrm{E} \max _{0 \leq k \leq n} S_{k}=\sqrt{\frac{2 n}{3 \pi}}-c+\frac{1}{5} \sqrt{\frac{2}{3 \pi}} n^{-1 / 2}+O\left(n^{-3 / 2}\right),
$$

where a numerical evaluation gives the constant $c=0.297952 \ldots$..

It will be clear that coefficients of further lower-order terms could be calculated for Theorem 2, but it will be equally clear that the calculations quickly become very awkward.

We apply Theorem 2 to the average-case analysis of the following simple algorithm for obtaining short packings of $n$ rectangles into a semi-infinite strip of width 1 (rectangles have widths at most 1, they can not be rotated, and they can not overlap each other or the boundaries of the strip). The algorithm is illustrated in Figure 1.

Algorithm:

1. Stack the rectangles with widths exceeding $1 / 2$ along the left edge of the strip in order of decreasing width. Let $H_{1 / 2}$ denote the height attained by these rectangles.

2. Starting at height $H_{1 / 2}$, stack the remaining rectangles along the right edge of the strip in order of increasing width.

3. Slide the stack on the right down until it rests on the bottom of the strip, or a rectangle in the right stack comes in contact with a rectangle in the left stack, whichever comes first.

4. Repack the rectangles lying entirely above $H_{1 / 2}$ into two stacks, one against the left edge of the strip and the other along the right edge. Pack these rectangles in decreasing order of height, with the $i$-th rectangle being placed on the shorter of the two stacks created by the first $i-1$ rectangles.

Let $X_{i}, Y_{i} \leq 1$ be the width and height dimensions of rectangle $i$ and define

$$
\delta(y)=\sum_{1 / 2-y \leq X_{i} \leq 1 / 2} Y_{i}-\sum_{1 / 2<X_{i} \leq 1 / 2+y} Y_{i}, \quad 0 \leq y \leq 1 / 2 .
$$

It is not hard to verify that the height of the packing produced by the algorithm at the end of step 3 is $H_{1 / 2}+\max _{0 \leq y \leq 1 / 2} \delta(y)$, so the final height $H$ after step 4 is bounded by

$$
H \leq H_{1 / 2}+\frac{1}{2} \max _{0 \leq y \leq 1 / 2} \delta(y)+\frac{1}{2} .
$$




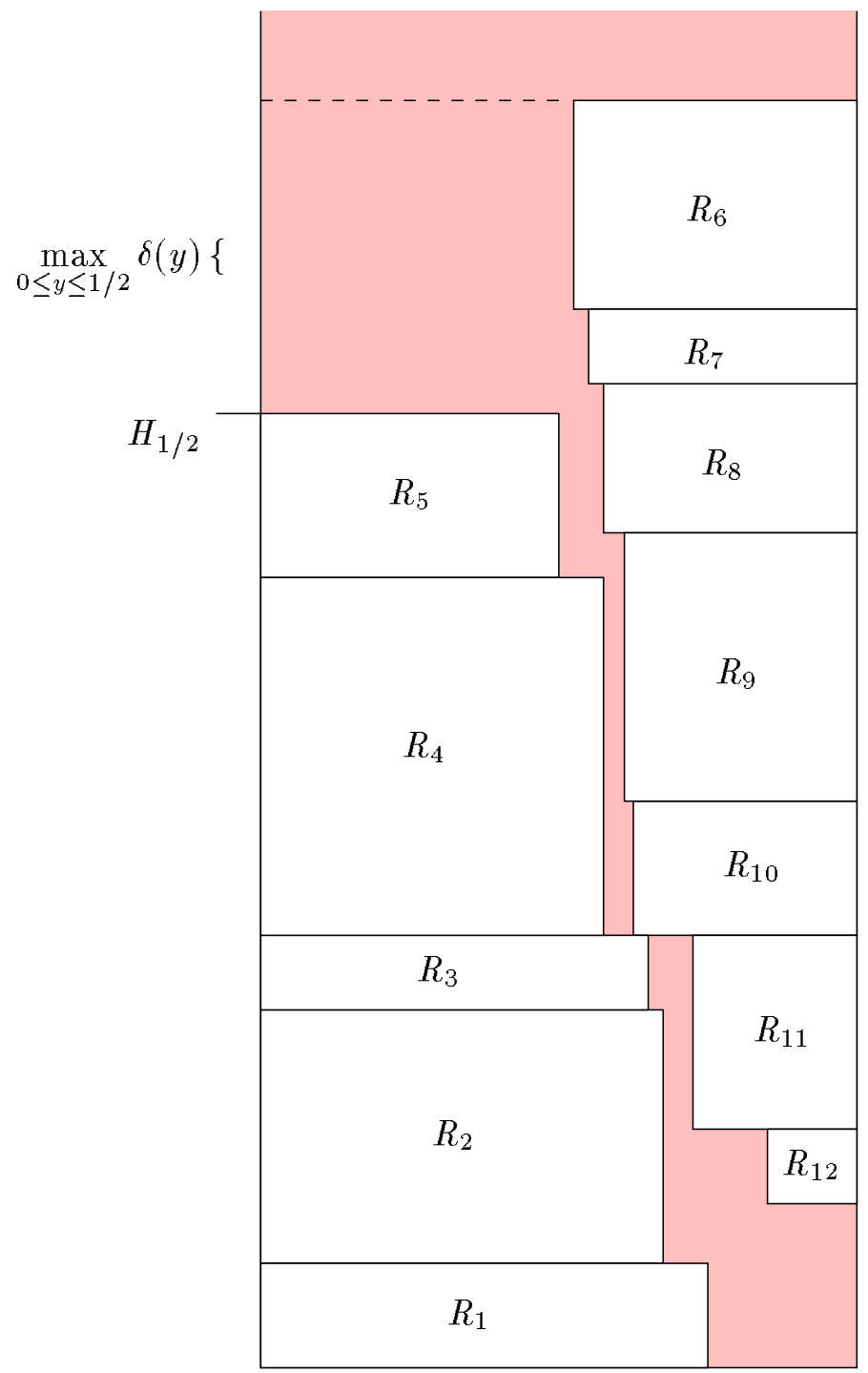

Figure 1: A packing after step 3 of rectangles $R_{1}, \ldots, R_{12}$. In the $4^{\text {th }}$ step $R_{6}$ would be moved over and put on top of $R_{5}$. 
For an average-case analysis, we adopt the uniform model in which the $X_{i}$ and $Y_{i}$ are $2 n$ independent uniform random draws from $[0,1]$. Then we obtain

$$
\mathrm{E} H \leq \frac{n}{4}+\frac{1}{2} \mathrm{E} \max _{0 \leq k \leq n} S_{k}+\frac{1}{2},
$$

since as observed in [3], $\max _{0 \leq y \leq 1 / 2} \delta(y)$ is equal in distribution to $\max _{0 \leq k \leq n} S_{k}$. We have $\mathrm{E} H=\frac{n}{4}+O\left(n^{1 / 2}\right)$ from classical results, but by substitution of Theorem 2 into (4), we obtain a much more precise estimate of the bound.

Theorem 3 In the uniform model, the expected height after step 3 is

$$
\frac{n}{4}+\sqrt{\frac{2 n}{3 \pi}}-c+\frac{1}{5} \sqrt{\frac{2}{3 \pi}} n^{-1 / 2}+O\left(n^{-3 / 2}\right),
$$

and so after the final step, step 4, the expected height is bounded by

$$
\mathrm{E} H \leq \frac{n}{4}+\sqrt{\frac{n}{6 \pi}}+\frac{1-c}{2}+\frac{1}{5 \sqrt{6 \pi}} n^{-1 / 2}+O\left(n^{-3 / 2}\right),
$$

with $c=.297952 \ldots$..

We remark further on this bound in the last section.

\section{Asymptotics of $I_{n}$}

We begin with a vital, but easily proved fact, viz., that the integral $I_{n}$ converges for all $n \geq 1$. To see this, it is enough to observe that

$$
\begin{aligned}
1-\prod_{j=1}^{n} \frac{x^{2}}{x^{2}+j^{2}} & =1-\prod_{j=1}^{n}\left[1+O\left(\frac{1}{x^{2}}\right)\right] \\
& =1-\left[1+O\left(\frac{1}{x^{2}}\right)\right]=O\left(\frac{1}{x^{2}}\right) .
\end{aligned}
$$

Before giving the proof of Theorem 1, we need a few lemmas.

Lemma 1 Let $j>-1$ and $a>0$. Then

$$
\int_{0}^{a} e^{-n w} w^{j} d w=\frac{\Gamma(j+1)}{n^{j+1}}+O\left(\frac{e^{-n a}}{n}\right) .
$$

Proof: Let $y=n w$. Then

$$
\begin{aligned}
\int_{0}^{a} e^{-n w} w^{j} d w & =\frac{1}{n^{j+1}} \int_{0}^{n a} e^{-y} y^{j} d y \\
& =\frac{1}{n^{j+1}}\left[\Gamma(j+1)-\int_{n a}^{\infty} e^{-y} y^{j} d y\right],
\end{aligned}
$$


and since

$$
\frac{d}{d x}\left[\int_{x}^{\infty} e^{-y} y^{j} d y\right]=-e^{-x} x^{j} \sim \frac{d}{d x}\left[e^{-x} x^{j}\right],
$$

as $x \rightarrow \infty$, we have, by l'Hospital's rule,

$$
\int_{n a}^{\infty} e^{-y} y^{j} d y \sim e^{-n a}(n a)^{j}
$$

as $n \rightarrow \infty$, which on substitution into (6) gives (5).

Lemma 2 Let $f_{n}(z), n \geq 1$, and $f(z)$ be analytic for $|z|<r$, and let $f_{n}(0)=$ $f(0)=0$ and $f^{\prime}(0) \neq 0$. Furthermore, let $f_{n}(z)$ converge uniformly to $f(z)$ for $|z|<r$. Then

(i) there exist $r_{1}, r_{2}>0$ and $N>0$, such that for $n>N, f(z)$ and $f_{n}(z)$ are univalent for $|z|<r_{1}$. The functions $z=g(w), z=g_{n}(w)$, which are the respective inverses of $w=f(z), w=f_{n}(z)$, are univalent for $|w|<r_{2}$. In addition, $g_{n}(w)$ converges uniformly to $g(w)$ for $|w|<r_{2}$.

(ii) there exists a $\kappa>0$ such that

$$
\left|g_{n}(w)-\sum_{k=0}^{m} \frac{g_{n}^{(k)}(0) w^{k}}{k !}\right| \leq \kappa\left(\frac{|w|}{r_{2}}\right)^{m+1},|w|<\frac{r_{2}}{2}, n>N, m \geq 0 .
$$

Proof: Part (i) follows from a careful examination of the inverse function theorem for analytic functions applied to the sequence $\left\{f_{n}(z)\right\}$. Part (ii) then follows from the Cauchy estimate for the coefficients of the power series for $g_{n}(w)$. We omit the details.

Let

$$
\begin{aligned}
& w_{n}(z)=\frac{1}{n} \sum_{j=1}^{n} \ln \left(1+\frac{j^{2}}{n^{2}} z\right), n \geq 1, \\
& w_{\infty}(z)=\int_{0}^{1} \ln \left(1+x^{2} z\right) d x,
\end{aligned}
$$

where $\ln$ is interpreted to be the principal value of the logarithm. Thus, $w_{n}(z)$ and $w_{\infty}(z)$ are analytic in the region $D$ defined as the complex plane minus the slit $[-\infty,-1]$. The functions $w_{n}(z)$ and $w_{\infty}(z)$ play a critical role in the proof of Theorem 1. In the next lemma, we collect various properties of these functions.

Lemma 3 The functions $w_{n}(z), w_{\infty}(z)$ satisfy the following:

(i) $\lim _{n \rightarrow \infty} w_{n}(z)=w_{\infty}(z), z \in D$;

(ii) $w_{n}(z)$ and $w_{\infty}(z)$ are strictly increasing for $0 \leq z<\infty ; w_{n}(0)=w_{\infty}(0)=0$, and $\lim _{z \rightarrow \infty} w_{n}(z)=\lim _{z \rightarrow \infty} w_{\infty}(z)=\infty$;

(iii) for $0<r<1, \lim _{n \rightarrow \infty} w_{n}(z)=w(z)$ uniformly for $|z|<r$;

(iv) $w_{\infty}^{\prime}(0)=1 / 3$. 
Proof: Part (i) follows from the fact that $w_{n}(z)$ is the Riemann sum for $w_{\infty}(z)$ and part (ii) follows from the fact that $\ln (1+z)$ is strictly increasing for $0 \leq$ $z<\infty$, with $\ln (1+0)=0$ and $\lim _{z \rightarrow \infty} \ln (1+z)=\infty$.

To verify (iii), write

$$
\left|w_{\infty}(z)-w_{n}(z)\right| \leq \sum_{j=1}^{n} \int_{(j-1) / n}^{j / n}\left|\ln \left(1+x^{2} z\right)-\ln \left(1+\frac{j^{2}}{n^{2}} z\right)\right| d x
$$

and observe that, for $\left|z_{1}\right|,\left|z_{2}\right|<r$,

$$
\left|\ln \left(1+z_{1}\right)-\ln \left(1+z_{2}\right)\right|=\left|\int_{z_{1}}^{z_{2}} \frac{d z}{1+z}\right| \leq \frac{\left|z_{2}-z_{1}\right|}{1-r} .
$$

Substituting $x^{2} z$ for $z_{1}$ and $\frac{j^{2}}{n^{2}} z$ for $z_{2}$, we obtain from (9) and (10)

$$
\left|w_{\infty}(z)-w_{n}(z)\right| \leq \frac{r}{1-r} \sum_{j=1}^{n} \int_{(j-1) / n}^{j / n}\left|x^{2}-\frac{j^{2}}{n^{2}}\right| d x \leq \frac{2 r}{1-r} \frac{1}{n}, \quad|z| \leq r,
$$

which proves (iii).

Differentiation gives

$$
w_{\infty}^{\prime}(z)=\int_{0}^{1} \frac{x^{2}}{1+x^{2} z} d x, \quad x \in D
$$

and in particular, $w_{\infty}^{\prime}(0)=\int_{0}^{1} x^{2} d x=1 / 3$, as desired for part (iv).

From the power series expansion for $\ln (1+z)$ we get

$$
w_{n}(z)=\sum_{k=1}^{\infty}(-1)^{k-1} A_{n k} z^{k}, \quad|z|<1
$$

with

$$
A_{n k}=\frac{1}{k n^{2 k+1}} \sum_{j=1}^{n} j^{2 k} .
$$

In the sequel, we only need $A_{n 1}, A_{n 2}$ which we rename as $A_{n}$ and $B_{n}$. Formulas for $A_{n}$ and $B_{n}$ are well known and yield

$$
\begin{aligned}
& A_{n}=\frac{1}{3}+\frac{1}{2 n}+O\left(\frac{1}{n^{2}}\right) \\
& B_{n}=\frac{1}{10}+O\left(\frac{1}{n}\right) .
\end{aligned}
$$

Proof of Theorem 1: Rewrite (1) as

$$
I_{n}=\int_{0}^{\infty}\left(1-\exp \left[-\sum_{j=1}^{n} \ln \left(1+\frac{j^{2}}{x^{2}}\right)\right]\right) d x .
$$


In terms of $z=n^{2} / x^{2}$, this becomes

$$
I_{n}=\frac{n}{2} \int_{0}^{\infty}\left[1-e^{-n w_{n}(z)}\right] z^{-3 / 2} d z
$$

where $w_{n}(z)$ is given by $(7)$. Integration by parts then yields

$$
I_{n}=n^{2} \int_{0}^{\infty} z^{-1 / 2} e^{-n w_{n}(z)} \frac{d w_{n}}{d z} d z .
$$

Let $z=z_{n}(w)$ be the inverse of $w=w_{n}(z)$. By Lemma 3(ii), $z_{n}(w)$ is defined for $0 \leq w<\infty$, and so (18) may be rewritten

$$
I_{n}=n^{2} \int_{0}^{\infty}\left[z_{n}(w)\right]^{-1 / 2} e^{-n w} d w
$$

The power series for $z_{n}$ can be computed by inverting the power series in (12) for $w_{n}(z)$. Thus, we can conclude from (12) and Lemmas 2 and 3 that, for some $0<a<1$,

$$
z_{n}(w)=\frac{w}{A_{n}}+\frac{B_{n}}{A_{n}^{3}} w^{2}+O\left(w^{3}\right), 0 \leq w \leq a,
$$

with the constant factor hidden in the O-term being the same for all $n$, up to a factor of $\left(1+O\left(n^{-1}\right)\right.$. Taking the square root of $(20)$, we find, for $0 \leq w \leq a$,

$$
z_{n}^{-1 / 2}=A_{n}^{1 / 2} w^{-1 / 2}-\frac{B_{n}}{2 A_{n}^{3 / 2}} w^{1 / 2}+O\left(w^{3 / 2}\right),
$$

where $w^{1 / 2} \geq 0$, and the hidden constant factor is still uniform in $n$.

We now rewrite (19) as

$$
I_{n}=\int_{0}^{a} K(w) d w+\int_{a}^{\infty} K(w) d w
$$

where

$$
K(w)=n^{2}\left[z_{n}(w)\right]^{-1 / 2} e^{-n w} .
$$

From Lemma 2 we obtain that $z_{n}(w)$ increases in both $n$ and $w$, for $w \geq 0$. Hence, for large $n$,

$$
\begin{aligned}
\int_{a}^{\infty} K(w) d w & \leq n^{2}\left[z_{n}(a)\right]^{-1 / 2} \int_{a}^{\infty} e^{-n w} d w \\
& \leq n\left[z_{\infty}(a)\right]^{-1 / 2} e^{-n a} .
\end{aligned}
$$

From (14), (15), and (21), we get, for $0 \leq w \leq a$,

$$
z_{n}^{-1 / 2}(w)=\left[3^{-1 / 2}+\frac{3^{1 / 2}}{4 n}+O\left(\frac{1}{n^{2}}\right)\right] w^{-1 / 2}-\left[\frac{3^{3 / 2}}{20}+O\left(\frac{1}{n}\right)\right] w^{1 / 2}+O\left(w^{3 / 2}\right),
$$


the hidden constant factor in $O\left(w^{3 / 2}\right)$ being independent of $n$. Combining this estimate with Lemma 1, we get

$$
\int_{0}^{a} K(w) d w=n^{2}\left[\frac{3^{-1 / 2} \Gamma\left(\frac{1}{2}\right)}{n^{1 / 2}}+\frac{3^{1 / 2} \Gamma\left(\frac{1}{2}\right)}{4 n^{3 / 2}}-\frac{3^{3 / 2} \Gamma\left(\frac{3}{2}\right)}{20 n^{3 / 2}}+O\left(\frac{1}{n^{5 / 2}}\right)\right] .
$$

Finally, we insert $\Gamma\left(\frac{1}{2}\right)=\sqrt{\pi}$ and $\Gamma\left(\frac{3}{2}\right)=\frac{1}{2} \sqrt{\pi}$ into (25), then add (25) to (23) and obtain the result of Theorem 1 .

\section{$3 \quad \operatorname{Emax}_{0 \leq k \leq n} S_{k}$ : Explicit Formulas}

The density of the position $S_{n}$ of the random walk is well known [4] and given by

$$
f_{n}(x)=\frac{1}{2^{n}(n-1) !} \sum_{j=0}^{n}(-1)^{j}\left(\begin{array}{l}
n \\
j
\end{array}\right)\left([x+(n-2 j)]^{+}\right)^{n-1},
$$

where as usual $y^{+}:=\max (y, 0)$. The distribution of the maximum of the random walk is given by the Pollaczek-Spitzer identity [6]. For the first moment we have

$$
E\left[\max _{0 \leq k \leq n} S_{k}\right]=\sum_{k=1}^{n} E\left[S_{k}^{+}\right] / k .
$$

To evaluate $\mathrm{E} S_{k}^{+}$using (26), the following combinatorial identities will be useful.

Lemma 4 For $n \geq 0$, we have

$$
\begin{aligned}
n ! & =(-1)^{n} \sum_{j=0}^{n}(-1)^{j}\left(\begin{array}{l}
n \\
j
\end{array}\right) j^{n} \\
\frac{n}{2}(n+1) ! & =(-1)^{n} \sum_{j=0}^{n}(-1)^{j}\left(\begin{array}{c}
n \\
j
\end{array}\right) j^{n+1} .
\end{aligned}
$$

Proof: For any function $F(x)$ defined for all real $x$, let $\Delta F(x):=F(x+1)-$ $F(x)$. An induction argument establishes

$$
\Delta^{n} F(x)=(-1)^{n} \sum_{j=0}^{n}(-1)^{j}\left(\begin{array}{l}
n \\
j
\end{array}\right) F(x+j) .
$$

Also by induction, one proves in particular that

$$
\Delta^{n}\left(x^{n}\right)=n !, \quad \Delta^{n}\left(x^{n+1}\right)=(n+1) ! x+\frac{n}{2}(n+1) ! .
$$

In (28), let $F(x)$ be $x^{n}$ then $x^{n+1}$, and put $x=0$. The lemma follows from (28) and (29). 
Returning now to the calculation of $\mathrm{E}_{k}^{+}$, write

$$
\mathrm{E} S_{n}^{+}=\int_{0}^{n} x f_{n}(x) d x=\frac{1}{2^{n}(n-1) !} \sum_{j=0}^{n}(-1)^{j}\left(\begin{array}{c}
n \\
j
\end{array}\right) I_{n j}
$$

where

$$
I_{n j}=\int_{\max (0,2 j-n)}^{n}(x-[2 j-n])^{n-1} x d x .
$$

To compute $I_{n j}$, let $y=x-[2 j-n]$ and consider the following cases.

Case 1. $n / 2 \leq j \leq n$. Then

$$
\begin{aligned}
I_{n j} & =\int_{0}^{2(n-j)} y^{n-1}(y+2 j-n) d y \\
& =-\frac{2^{n+1}(n-j)^{n+1}}{n(n+1)}+2^{n}(n-j)^{n} .
\end{aligned}
$$

Case 2. $0 \leq j<n / 2$. Then

$$
\begin{aligned}
I_{n j} & =\int_{n-2 j}^{2(n-j)} y^{n-1}(y+2 j-n) d y \\
& =-\frac{2^{n+1}(n-j)^{n+1}}{n(n+1)}+2^{n}(n-j)^{n}+\frac{1}{n(n+1)}(n-2 j)^{n+1} .
\end{aligned}
$$

Now substitute (32) and (33) into (30) and let $k=n-j$. We get

$$
\begin{aligned}
\mathrm{E}_{n}^{+}= & -\frac{2}{(n+1) !}(-1)^{n} \sum_{j=0}^{n}(-1)^{j}\left(\begin{array}{l}
n \\
j
\end{array}\right) j^{n+1}+\frac{(-1)^{n}}{(n-1) !} \sum_{j=0}^{n}(-1)^{j}\left(\begin{array}{l}
n \\
j
\end{array}\right) j^{n} \\
& +\frac{2(-1)^{n}}{(n+1) !} \sum_{n / 2 \leq j \leq n}(-1)^{j}\left(\begin{array}{l}
n \\
j
\end{array}\right)(j-n / 2)^{n+1} .
\end{aligned}
$$

By Lemma 4, the first two terms on the right-hand side of (34) cancel, so we are left with

$$
\mathrm{E} S_{n}^{+}=\frac{2(-1)^{n}}{(n+1) !} \sum_{n / 2 \leq j \leq n}(-1)^{j}\left(\begin{array}{l}
n \\
j
\end{array}\right)(j-n / 2)^{n+1} .
$$

This sum does not seem to simplify, and adding the summation in (27) does not help. But to make an asymptotic analysis easier, we can convert (35) into an integral form using Rice's method as follows.

Let $\mathrm{E} S_{k}^{+}=\frac{2}{k+1} D_{k}$ to allow neater expressions. Then

$$
D_{k}=\frac{(-1)^{k}}{k !} \sum_{k / 2 \leq j \leq k}(-1)^{j}\left(\begin{array}{l}
k \\
j
\end{array}\right)(j-k / 2)^{k+1}=\frac{1}{2 \pi i} \oint_{C_{N}} \frac{(s-k / 2)^{k+1}}{s(s-1) \cdots(s-k)} d s
$$


where $C_{N}$ is the contour shown in figure 2 , that consists of a half circle with the diameter boundary passing through the real value $k / 2$. The integral representation follows from Cauchy's theorem, since the only singularities of the integrand in (36) are poles, and for $N>k$ the residues at the poles inside $C_{N}$ are just those terms of the sum.

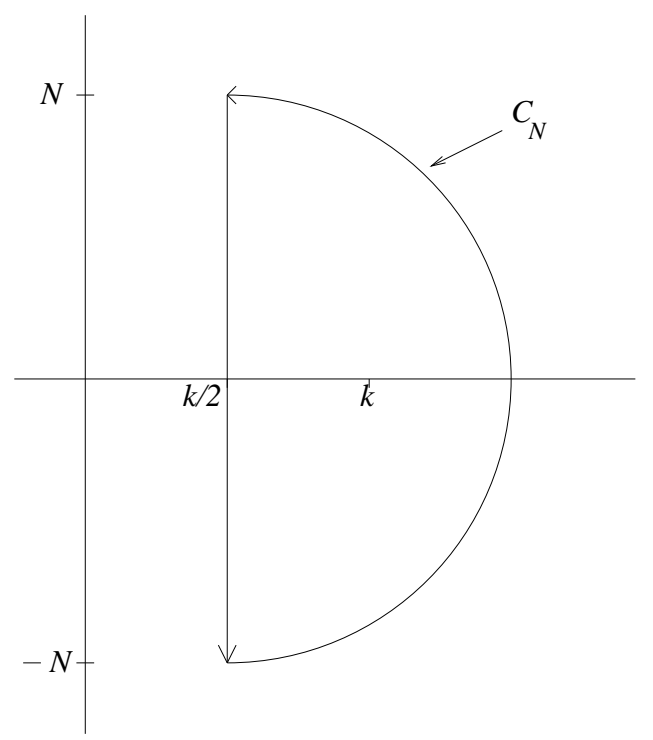

Figure 2: Integration contour for equation (36)

Let $F_{k}(s)$ denote the integrand in (36). By one more application of Cauchy's theorem, we may replace $F_{k}(s)$ by $F_{k}(s)-1$ in (36). A simple calculation shows that $F_{k}(s)-1=O\left(|s|^{-2}\right)$ as $s \rightarrow \infty$. Hence, as $N \rightarrow \infty$, the contribution of the half circle to the integral vanishes, and we conclude that

$$
D_{k}=-\frac{1}{2 \pi i} \int_{k / 2-i \infty}^{k / 2+i \infty}\left[\frac{(s-k / 2)^{k+1}}{s(s-1) \cdots(s-k)}-1\right] d s,
$$

We can simplify (37) as follows, with the resulting form depending on the parity of $k$,

$$
\begin{aligned}
D_{2 n} & =\frac{1}{\pi} \int_{0}^{\infty}\left[1-\prod_{j=1}^{n} \frac{x^{2}}{x^{2}+j^{2}}\right] d x, \quad n \geq 1, \\
D_{2 n-1} & =\frac{1}{\pi} \int_{0}^{\infty}\left[1-\prod_{j=1}^{n} \frac{x^{2}}{x^{2}+(j-1 / 2)^{2}}\right] d x, \quad n \geq 1 .
\end{aligned}
$$




\section{$4 \quad \operatorname{Emax}_{0 \leq k \leq n} S_{k}:$ Asymptotics}

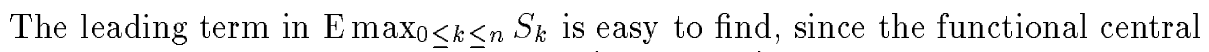
limit theorem states that the process $\left(\frac{S_{n t}}{\sigma \sqrt{n}}, t \geq 0\right)$ converges in distribution to standard Brownian motion ( $\sigma^{2}=1 / 3$ is the variance of the uniform step distribution on $[-1,+1])$. The probability that the maximum of standard Brownian motion starting at the origin exceeds $x$ in the time interval $[0, t]$ is given by $2[1-\Phi(x / \sqrt{t})][4$, p. 175$]$, so we obtain

$$
P\left(\max _{0 \leq k \leq n} S_{k}>y\right) \sim \frac{2}{2 \pi} \int_{\frac{y}{\sigma \sqrt{n}}}^{\infty} e^{-x^{2} / 2} d x,
$$

as $n \rightarrow \infty$. An integration over $0 \leq y \leq \infty$ then shows that

$$
\frac{\operatorname{E} \max _{0 \leq k \leq n} S_{k}}{\sqrt{n}} \rightarrow \sqrt{\frac{2}{3 \pi}}
$$

as $n \rightarrow \infty$, the desired constant. This constant will be verified below in an analysis that also yields lower order terms.

Note that formula (38) is $I_{n} / \pi$, where the asymptotics of $I_{n}$ are given in Theorem 1. An analysis of (39) leads us to a similar asymptotic result, as shown below.

Lemma 5 We have

$$
D_{k}=\frac{1}{2 \sqrt{6 \pi}} k^{3 / 2}+\frac{7}{40} \sqrt{\frac{3}{2 \pi}} k^{1 / 2}+O\left(k^{-1 / 2}\right) .
$$

Proof: Theorem 1 proves (40) for even $k$. For odd $k$ we need the asymptotics of

$$
J_{n}=\int_{0}^{\infty}\left[1-\prod_{j=1}^{n} \frac{x^{2}}{x^{2}+(j-1 / 2)^{2}}\right] d x .
$$

We prove next that

$$
J_{n}=\sqrt{\frac{\pi}{3}} n^{3 / 2}-\frac{3 \sqrt{3 \pi}}{40} n^{1 / 2}+O\left(n^{-1 / 2}\right) .
$$

The proof of (42) mimics that of Theorem 1 . We replace $j$ by $j-1 / 2$ in (16), so that now

$$
w_{n}(z)=\frac{1}{n} \sum_{j=1}^{n} \ln \left[1+\frac{(j-1 / 2)^{2}}{n^{2}} t\right] .
$$

With this replacement, Lemma 3 still holds, but we must now remove the term $\frac{1}{2 n}$ from the asymptotic formula for $A_{n}$ given in (14). (The asymptotic formula for $B_{n}$ remains the same.) This in turn forces the removal of the terms $\frac{3^{1 / 2}}{4 n}$ and 
$\frac{3^{1 / 2} \Gamma(1 / 2)}{4 n^{3 / 2}}$ from (24) and (25), respectively. Carrying out these changes yields (42) as desired.

Now write $D_{k}=\frac{1}{\pi} J_{\frac{k+1}{2}}$ for odd $k$. Letting $n=(k+1) / 2$ in (42) and using

$$
\begin{aligned}
& (k+1)^{1 / 2}=k^{1 / 2}+O\left(k^{-1 / 2}\right) \\
& (k+1)^{3 / 2}=k^{3 / 2}+\frac{3}{2} k^{1 / 2}+O\left(k^{-1 / 2}\right),
\end{aligned}
$$

we obtain (40).

By Lemma 5, we now have

$$
\frac{\mathrm{E} S_{k}^{+}}{k}=\frac{2 D_{k}}{k(k+1)}=\frac{1}{\sqrt{6 \pi}} k^{-1 / 2}+\frac{1}{20 \sqrt{6 \pi}} k^{-3 / 2}+\theta_{k},
$$

where the remainder term satisfies $\theta_{k}=O\left(k^{-5 / 2}\right)$.

Proof of Theorem 2: $\quad$ Express E $\max _{0 \leq k \leq n} S_{k}$ as the sum in (27), and then substitute for $\mathrm{E}_{k}^{+} / k$ from (43) to obtain

$$
\mathrm{E} \max _{0 \leq k \leq n} S_{k}=\sum_{k=1}^{n} \frac{\mathrm{E} S_{k}^{+}}{k}=\frac{1}{\sqrt{6 \pi}} \sum_{k=1}^{n} k^{-1 / 2}+\frac{1}{20 \sqrt{6 \pi}} \sum_{k=1}^{n} k^{-3 / 2}+\Theta_{n} .
$$

where $\Theta_{n}:=\sum_{1 \leq k \leq n} \theta_{k}$. Now apply the Euler-Maclaurin formula to the last two sums in (44). Standard manipulations show that (see e.g. [7, Ex. 3.2, p. 292])

$$
\begin{aligned}
& \sum_{k=1}^{n} k^{-1 / 2}=2 n^{1 / 2}+\zeta(1 / 2)+n^{-1 / 2} / 2+O\left(n^{-3 / 2}\right) \\
& \sum_{k=1}^{n} k^{-3 / 2}=\zeta(3 / 2)-2 n^{-1 / 2}+O\left(n^{-3 / 2}\right),
\end{aligned}
$$

where $\zeta(s)=\sum_{k \geq 1} k^{-s}, \Re(s)>1$, is the Riemann zeta function, and where, by analytic continuation (see e.g. [1], formula 23.2.9),

$$
\zeta(1 / 2)=\lim _{n \rightarrow \infty}\left(\sum_{1 \leq k \leq n} k^{-1 / 2}-2 n^{1 / 2}\right) .
$$

Substituting (45) and (46) into (44), we get

$$
\mathrm{E} \max _{0 \leq k \leq n} S_{k}=\sqrt{\frac{2}{3 \pi}} n^{1 / 2}-c_{n}+\frac{1}{5} \sqrt{\frac{2}{3 \pi}} n^{-1 / 2}+O\left(n^{-3 / 2}\right),
$$

where

$$
c_{n}=-\left[\frac{\zeta(1 / 2)}{\sqrt{6 \pi}}+\frac{\zeta(3 / 2)}{20 \sqrt{6 \pi}}+\Theta_{n}\right]
$$


Now $\theta_{k}=O\left(k^{-5 / 2}\right)$ so an easy application of the Euler-Maclaurin formula gives $\sum_{k>n} \theta_{k}=O\left(n^{-3 / 2}\right)$, and therefore $\Theta_{\infty}=\Theta_{n}+O\left(n^{-3 / 2}\right)$ and $c:=c_{\infty}=$ $c_{n}+O\left(n^{-3 / 2}\right)$. Thus, we can replace $c_{n}$ by $c$ in (47), since the error introduced in doing so is of the same order as the error term already in (47). The limit $n \rightarrow \infty$ in (44) yields

$$
\Theta_{\infty}=\sum_{k \geq 1} \theta_{k}=\sum_{k \geq 1}\left(\frac{\mathrm{E} S_{k}^{+}}{k}-\frac{k^{-1 / 2}}{\sqrt{6 \pi}}-\frac{k^{-3 / 2}}{20 \sqrt{6 \pi}}\right),
$$

so on substitution into the limit $n \rightarrow \infty$ of (48), we get

$$
c=-\left[\frac{\zeta(1 / 2)}{\sqrt{6 \pi}}+\frac{\zeta(3 / 2)}{20 \sqrt{6 \pi}}+\sum_{k \geq 1}\left(\frac{\mathrm{E} S_{k}^{+}}{k}-\frac{k^{-1 / 2}}{\sqrt{6 \pi}}-\frac{k^{-3 / 2}}{20 \sqrt{6 \pi}}\right)\right],
$$

where $\mathrm{E} S_{k}^{+}$is determined by (35). Equation (49) and straightforward computations show that $c=.297952 \ldots$ is precise to 6 decimal digits, so Theorem 2 is proved.

Remark. As might be expected, the constant $c$ can be computed to much greater accuracy than that given above. With Maple doing the computations, we demonstrated this, basing our numerical method on Romberg acceleration (see e.g. $[5, \S 11.12]$ ). Our results indicate that $\left|c-c_{n}\right| \approx \frac{11}{1680} \frac{n^{-3 / 2}}{\sqrt{6 \pi}}$, and that

$$
c=0.2979521902800477642 \ldots,
$$

with an error of at most $10^{-19}$.

\section{Final Remarks}

It would be interesting to know whether (4) is tight, or asymptotically so, within a constant term, i.e., does there exist a constant such that for any algorithm, $\frac{n}{4}+\frac{1}{2} \mathrm{E} \max _{0 \leq k \leq n} S_{k}$ is within that constant of the expected packing height for all $n$ sufficiently large? Note that this would follow if it could be shown that, with a probability that tends to 1 as $n \rightarrow \infty$, all rectangles above the point where the downward slide of the right stack is stopped (see Figure 1) have a width at least $1 / 3$.

The rectangle packing problem is an extension of the square packing problem studied earlier in [2], in which square sizes are determined by uniform random draws from $[0,1]$. For squares, the $Y_{i}$ in (2) need to be changed to $X_{i}$. The problem is again to find the expected maximum positive excursion of an $n$-step random walk starting at the origin, but the random walk is now biased and nonhomogeneous. It is known that the expected packing height is

$$
\mathrm{E} H=\frac{3 n}{8}+\Theta\left(n^{1 / 3}\right)
$$


where the first term is $\mathrm{E} H_{1 / 2}$ and the second term is the expected maximum of the random walk. However, the techniques in [2] shed no light on the hidden multiplicative constant (much less on lower-order terms).

\section{References}

[1] M. Abramowitz and I. A. Stegun, Handbook of Mathematical Functions, Dover Publications, New York, 1972.

[2] E. G. Coffman, Jr. and J. C. Lagarias. A Probabilistic Analysis of Square Packing. SIAM J. Comput., 18:166-185, 1989.

[3] E. G. Coffman, Jr. and P. W. Shor. Packing in two dimensions: Asymptotic average-case analysis of algorithms. Algorithmica, 9(3):253-277, March 1993.

[4] W. Feller, An Introduction to Probability Theory and its Applications, Vol. II, 2nd Ed., John Wiley \& Sons, New York (1971).

[5] P. Henrici, Applied and Computational Complex Analysis, Vol. II, John Wiley \& Sons, New York (1977).

[6] M. Hofri, Analysis of Algorithms: Computational Methods $\&$ Mathematical Tools, Oxford University Press, New York (1995).

[7] F. W. J. Olver. Asymptotics and Special Functions, Academic Press, New York (1974). 


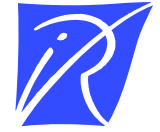

Unité de recherche INRIA Lorraine, Technopôle de Nancy-Brabois, Campus scientifique, 615 rue du Jardin Botanique, BP 101, 54600 VILLERS LĖS NANCY

Unité de recherche INRIA Rennes, Irisa, Campus universitaire de Beaulieu, 35042 RENNES Cedex Unité de recherche INRIA Rhône-Alpes, 655, avenue de l'Europe, 38330 MONTBONNOT ST MARTIN

Unité de recherche INRIA Rocquencourt, Domaine de Voluceau, Rocquencourt, BP 105, 78153 LE CHESNAY Cedex

Unité de recherche INRIA Sophia-Antipolis, 2004 route des Lucioles, BP 93, 06902 SOPHIA-ANTIPOLIS Cedex

Éditeur

INRIA, Domaine de Voluceau, Rocquencourt, BP 105, 78153 LE CHESNAY Cedex

(France)

http://www.inria.fr

ISSN 0249-6399 